\title{
LC-MS/MS-based reference intervals for hair cortisol in healthy children
}

\author{
Ineke de Kruijff ${ }^{\mathrm{a}}$, Gerard Noppe ${ }^{\mathrm{b}}$, Noera Kieviet ${ }^{\mathrm{c}}$, Vandhana Choenni ${ }^{\mathrm{d}}$, \\ Mijke P Lambregtse-van den Berg ${ }^{\mathrm{d}}$, Dominique G.A. Begijn ${ }^{\mathrm{e}}$, Ellen Tromp ${ }^{\mathrm{f}}$, Kristien Dorst ${ }^{g}$, \\ Elisabeth F.C. van Rossum ${ }^{b}$, Yolanda. B. de Rijke ${ }^{g, 1}$, Erica L.T. van den Akker ${ }^{\mathrm{h},}, 1$
}

${ }^{\text {a }}$ Department of Pediatrics, St Antonius Hospital Nieuwegein, the Netherlands

${ }^{\mathrm{b}}$ Department of Internal Medicine, Division of Endocrinology, Erasmus MC, Rotterdam, the Netherlands

${ }^{\mathrm{c}}$ Department of Pediatrics, Amsterdam University Medical Center, Amsterdam, the Netherlands

${ }^{\mathrm{d}}$ Departments of Psychiatry and Child and Adolescent Psychiatry/Psychology, Erasmus MC, Rotterdam, the Netherlands

${ }^{\mathrm{e}}$ University of Utrecht, Utrecht, The Netherlands

${ }^{\mathrm{f}}$ Department of Epidemiology and Statistics, St Antonius Hospital Nieuwegein, the Netherlands

${ }^{\mathrm{g}}$ Department of Clinical Chemistry, Erasmus MC, Rotterdam, the Netherlands

${ }^{\text {h }}$ Department of Pediatrics, Sophia Children's Hospital, Erasmus MC, Rotterdam, The Netherlands

\section{A R T I C L E I N F O}

\section{Keywords:}

Cortisol

Scalp hair

Reference ranges

Infant

Children

\begin{abstract}
A B S T R A C T
Background: Human scalp hair is a valuable matrix for determining long-term cortisol concentrations, with widespread applicability in clinical care as well as research. However, pediatric reference intervals are lacking. The aim of this cross-sectional study is to establish age-adjusted reference intervals for hair cortisol in children and to gain insight into hair growth velocity in children up to 2 years old.

Methods: A total of 625 healthy children were enrolled through recruitment in pregnancy, infant-welfare clinics, and school visits. Scalp hair cortisol levels were measured using liquid chromatography-tandem mass spectrometry. Age-adjusted reference intervals were established in children from birth to 18 years old. Hair growth velocity was determined in children $0-2$ years of age by measuring hair length at 4- to 10-week intervals. Results: Hair cortisol levels were high $(162.4 \mathrm{pg} / \mathrm{mg}, 2.5$ th-97.5th percentile: $28.8-961)$ after birth with a sharp fall in the first 3 months of life. This is followed by lower values until age 6 and then by graduated and subtle higher values to adult concentrations are reached at the age of 18 years $(3.0 \mathrm{pg} / \mathrm{mg}, 2.5$ th-97.5th percentile: $0.53-17.8$ ). Average hair growth velocity measured in $\mathrm{mm} / \mathrm{month}$ was significantly lower in infants $0-6 \mathrm{months}$ of age compared to children $12-24$ months (3.5 versus $9.4, \mathrm{P}<0.001$ ).

Conclusions: This is the first study to provide age-adjusted reference intervals for hair cortisol in children from $0-18$ years. Higher hair cortisol concentrations in infants might be explained by the significantly lower hair growth rate in the first year of life. The establishment of pediatric hair cortisol reference ranges broadens the potential applications of this biomarker in pediatric clinical care.
\end{abstract}

\section{Introduction}

Measurement of endogenously produced cortisol is a cornerstone for diagnosis and monitoring of a broad range of diseases. Routinely used matrices for cortisol quantification include serum, urine and saliva. However, interpretation of diagnostic tests for cortisol is complicated by the pulsatile excretion, the naturally occurring circadian rhythm and the maximum of $24 \mathrm{~h}$ of measurement. Over the past decade, cortisol measurement of human scalp hair as a long-term biomarker of the hypothalamus-pituitary-adrenal (HPA) axis (Russell et al., 2018; Wester and Van Rossum, 2015) has become increasingly popular. Hair use offers unique benefits and characteristics, which include non-invasive sampling and a long-term retrospective reflection of cumulative hormone concentrations. These features make the use of scalp hair attractive for both clinical practice and research.

Studies have associated increased scalp hair cortisol with obesity (Noppe et al., 2016; Papafotiou et al., 2017) and, in adults, with metabolic syndrome (Stalder et al., 2013) and cardiovascular disease

\footnotetext{
Abbreviations: LC-MS/MS, liquid chromatography tandem mass spectrometry; GC, glucocorticoids; LLoQ, lower limit of quantification

* Corresponding author at: Department of Pediatrics, Sophia Children's Hospital, Erasmus MC, University Medical Center Rotterdam, Office Sp 1536 Wytemaweg 80, $3015 \mathrm{CN}$, Rotterdam, the Netherlands.

E-mail address: e.l.t.vandenakker@erasmusmc.nl (E.L.T. van den Akker).

${ }^{1}$ Shared last authors.
} 
(Manenschijn et al., 2013). Moreover, measurement of cortisol in hair may be an interesting tool as diagnostic test in Cushing's disease (Hodes et al., 2018; Savas et al., 2019) and is a promising tool for monitoring hydrocortisone treatment in adrenal insufficiency in both adults (Ibrahim and Van Uum, 2014) and children (Noppe et al., 2014). In addition, hair cortisol has been associated with mental health in adult literature; altered cortisol levels are found in chronic stress and anxiety disorders such as post-traumatic stress disorder (Stalder et al., 2017; Staufenbiel et al., 2013). In children, however, no significant relationship has been found between hair cortisol and anxiety or depression, and results in relation to stress and/or trauma exposure (Gray et al., 2018) have been surprisingly heterogeneous.

In adults, population-based reference ranges for hair cortisol measurement have been established in large cohort studies of healthy volunteers with liquid chromatography mass spectrometry (Abell et al., 2016; Greff et al., 2018; Staufenbiel et al., 2015; Wester et al., 2017). Limited studies of reference intervals of cortisol concentration in children have been published (Dettenborn et al., 2012; Karlen et al., 2013; Rippe et al., 2016); no extended studies of reference intervals using the state-of-the-art method of liquid chromatography-tandem-mass spectrometry (LC-MS/MS)(Greff et al., 2018) have been published. It is generally accepted that hair in adults grows at a fairly constant rate of approximately $10 \mathrm{~mm}$ per month (Saitoh et al., 1969), meaning that the first centimeter proximal to the scalp is assumed to contain cortisol incorporated during the month prior to sampling. Studies of children's hair characteristics and hair growth patterns, needed for correct interpretation of hair cortisol concentration, are limited (Barth, 1987). A study in 20 children aged $3-9$ years showed a growth rate of $9.6 \mathrm{~mm}$ per month (Pecoraro et al., 1964). To our knowledge, studies on hair growth rate in infants aged $0-2$ years are non-existent.

In this study we assess hair growth velocity in children aged $0-2$ years, establish age-adjusted reference intervals for cortisol in healthy children aged 0-18years and determine the influence of covariates such as hair color and hair treatment.

\section{Materials and methods}

\subsection{Subjects}

In this study, data were analyzed from three groups of healthy children aged $0-18$ years who were recruited in pregnancy and infantwelfare clinics, during elementary and secondary school visits and at a pediatric (outpatient) clinic.

The first group consisted of newborns who participated in the control group of a non-randomized, prospective controlled study in the OLVG West Hospital in Amsterdam, the Netherlands (Hollanders et al., 2017; Kieviet et al., 2016). Included were infants whose mothers did not use psychotropic medication during pregnancy, who were admitted to the maternity ward or neonatal care unit and who had an expected hospital stay of at least $72 \mathrm{~h}$. Most infants who fulfilled these criteria were born by caesarean section. The study was approved by the medical ethics committees of the OLVG West Hospital and VU Medical Center in Amsterdam, the Netherlands. Written informed consent was obtained from parents of all participants. Study recruitment took place between February 2012 and August 2013.

The second group consisted of six-week-old infants from the INCAS study, a multicenter observational study on maternal parenting capacity and infant development in mothers with severe mental illness. Infants of healthy mothers in the control group were recruited during pregnancy at several midwifery practices in the Netherlands. The INCAS study was approved by the Erasmus MC medical research ethics committee, and written informed consent was obtained from all participating mothers (NL42662.078012). Study recruitment took place between February 2013 and December 2014.

The third group consisted of healthy children aged $0-18$ years recruited from the general population of the Netherlands for a cross- sectional observational study on steroid hormone concentrations in scalp hair during childhood, adolescence and pregnancy. Participants were enrolled through visits to infant-welfare centers and primary and secondary schools in Rotterdam and Woerden and surrounding areas. In addition, two groups of children visiting an outpatient clinic were asked to participate in this study: 1) 12-week-old infants at risk for pathological developmental dysplasia of the hip who tested negative with sonographic screening and 2) healthy siblings of patients visiting the outpatient clinic. The criteria of exclusion we used were chronic disease and systemic use of glucocorticoids (GC) or other medications affecting steroid metabolism. Additional criteria of exclusion used for children aged less than 6 months were prematurity (gestational age $<37$ weeks) and systemic maternal use of GC. This study was approved by the Medical Research Ethics Committee of Erasmus MC and followed the Helsinki declaration. Written consent was obtained from parents and children aged 12 and above, and informed assent was given by children less than 12 years. Study recruitment took place between January 2011 and April 2018.

\subsection{Determinants}

For a subset of children questionnaires were filled out either by parents/caretakers or by children themselves (if older than 12 years). These covered hair care characteristics, general health and use of medication. Information was obtained about washing frequency $(<1$, $1-2,3-4$, or $>4$ times per week), use of hair products on the day of sampling (yes/no, type of product: none, mousse, gel, wax, spray, other), frequent sweating of the scalp (yes/no), hair treatment (e.g. dyeing, bleaching, or perming) in the past three months, topical use of GC and current tobacco smoking status (yes/no). In children who did not fill out a questionnaire, hair treatment was set to" no" for children under the age of 10 years and current smoking status was set to" no" for those under the age of 12 years. Ethnicity was determined according to country of birth of the children and their parents, derived from the completed questionnaires. Ethnicity is divided into three groups: native Dutch, Western background and non-Western background. Ethnicities are determined based on a report of Statistics Netherlands (CBS.nl). An immigrant is defined as a person one or both of whose parents were not born in the Netherlands. A Western immigrant is defined as a person one or both of whose parents were born in one of the countries in Europe (excluding Turkey), North America and Oceania, or Indonesia or Japan. A non-Western immigrant is defined as a person one or both of whose parents were born in one of the countries in the continents of Africa, Latin America and Asia (excluding Indonesia and Japan) or Turkey (Vliegenthart et al., 2016). (Statistics Netherlands, StatLine, CBS 2018).

\subsection{Hair collection and preparation}

Hair locks were cut with small surgical scissors from the posterior vertex, as close to the scalp as possible. The posterior vertex has the lowest variation in repeated measurement (Noppe et al., 2015; Sauvé et al., 2007). The hair locks were taped to a paper form with the scalp end marked and then stored in envelopes at room temperature. In the children aged 4 years and up, the proximal $3 \mathrm{~cm}$ of hair was used for a single analysis. The strands of hair were cut into 1-cm segments. In the newborn infants, the total length of fetal hair, typically $1 \mathrm{~cm}$, was analyzed. In children up to 4 years, whose hair had not always grown to $3 \mathrm{~cm}$, at least the $1 \mathrm{~cm}$ of hair closest to the scalp was analyzed. Subsequently, hair samples were transferred to glass tubes, weighed (mg), washed by gently shaking them in LC-MS grade isopropanol at room temperature and left to dry for at least $48 \mathrm{~h}$. To determine the hair growth velocity in children up to 2 years, the place of cutting was marked and described in the logbook. A researcher, research nurse and trained student were trained to perform both the cutting and measurement. At a follow-up appointment 1-2.5 months later, the new- 
grown hair was measured with a measuring tape by the same person who had done the cutting.

\subsection{Hair Analysis}

Cortisol was extracted in $1.5 \mathrm{~mL}$ LC-MS grade methanol for $18 \mathrm{~h}$ at $25{ }^{\circ} \mathrm{C}$ in the presence of deuterated cortisol-d3, and it was subsequently cleaned using solid phase extraction. The Department of Clinical Chemistry uses an ISO 15189;2012-accredited LC-MS/MS method for hair cortisol analysis and participates in the international interlaboratory Round Robin since 2015 (Russell et al., 2015). The method is described in detail elsewhere (Noppe et al., 2015) and uses a Waters Xevo TQ-S system (Waters Corporation, Milford, MA, USA). Cortisol concentrations were reported in pg per mg hair. The inter-assay coefficient of variation for cortisol was $14.8 \%$. The intra-assay coefficient of variation for cortisol was $<11 \%$. The lower limit of quantification (LLoQ) of cortisol was $<1 \mathrm{pg} / \mathrm{mg}$. Measurements below LLoQ were excluded from statistical analyses.

\subsection{Statistical Analysis}

Statistical analyses were performed with SPSS Version 24 (IBM, New York, USA). Age-specific reference intervals for hair cortisol concentrations were estimated by analysis of 2.5 and 97.5 percentile curves via a semi-parametric model with Box-Cox transformation, which is available in the" Generalized Additive Models for Location, Scale and Shape" package (Rigby et al., 2005). To prevent overfitting, model fit was assessed using Akaike's" information criterion" and visual inspection. Hair cortisol concentrations were transformed to age-adjusted zscores for subsequent analyses. Associations of hair cortisol concentrations with hair characteristics, smoking, gender and ethnicity were assessed using linear regression models and applicable adjustments for gender and ethnicity were applied. As hair cortisol concentrations have a skewed distribution, log-transformation was applied to achieve a normal distribution, and hair cortisol concentrations were transformed to age-adjusted z-scores. Associations of hair growth velocity with the three age groups were assessed using one-way ANOVA.

\section{Results}

Cortisol concentrations in a total of 625 children aged $0-18$ years were measured to establish reference intervals. The general characteristics of participants are shown in Table 1 . The three groups of healthy children were significantly different in age $(P<0.001)$ and in ethnicity $(\mathrm{P}<0.001)$. The participants' ethnic background was mainly native Dutch (Caucasian) in the second $(71 \%)$ and third (80\%) groups and mainly immigrant with a Western origin (76\%) in the first group, in which $24 \%$ were of Caucasian ethnicity. The groups did not significantly differ in gender $(\mathrm{P}=0.079)$.

The results of hair cortisol levels by age are shown in Fig. 1. Reference intervals are defined as median and predicted $2.5^{\text {th }}$ and $97.5^{\text {th }}$ percentiles, as shown in Table 2.

Newborns' scalp hair shows high levels of cortisol after birth, with a sharp fall in the first 3 months followed by further lower levels to the age of 6 years and then by graduated and subtle higher values to adult levels. The variation of cortisol levels was smaller in adolescents than in preschool-aged children.

\subsection{HAIR GROWTH VELOCITY}

Fig. 2 shows the hair growth velocity in a subset $(n=62)$ of children in the $<2$ years age group. The average hair growth velocity in infants 0-6 months old was $3.5 \mathrm{~mm}$ per month (SD 2.1; $\mathrm{n}=27$ ). The hair growth velocity in children aged 6-12 months was $6.7 \mathrm{~mm}$ per month (SD 3.1; $\mathrm{n}=21$ ) and in children $12-24$ months, $9.4 \mathrm{~mm}$ per month (SD 1.6; $n=14$ ). The hair growth velocity is significantly
Table 1

Descriptive statistics of study participants $(n=625)$.

\begin{tabular}{|c|c|}
\hline \multicolumn{2}{|l|}{ Participants } \\
\hline Age, years $(n=625)$ & $5.0(\mathrm{SD} 6.0)$ \\
\hline Height,SDS $(n=422)$ & $0.11(\mathrm{SD} 1.04)$ \\
\hline Body mass index, SDS $(n=422)$ & $-0.12(\mathrm{SD} 1.08)$ \\
\hline Gender, girls/boys $(n=625)$ & $299 / 326(48 / 52 \%)$ \\
\hline \multicolumn{2}{|l|}{ Ethnicity $(n=482)$} \\
\hline - Native Dutch (Caucasian) & $349(72.4 \%)$ \\
\hline - Immigrant non western/western & $50 / 83(10.4 / 17.2 \%)$ \\
\hline \multicolumn{2}{|l|}{ (Hair) Characteristics } \\
\hline \multicolumn{2}{|l|}{ Hair color $(n=237)$} \\
\hline - Black & $20(8.4 \%)$ \\
\hline - Brown & $116(48.9 \%)$ \\
\hline - Blond & $87(36.7 \%)$ \\
\hline - Red & $14(5.9 \%)$ \\
\hline Hair dyed yes/no $(\mathrm{n}=578)^{*}$ & $5 / 573(1 / 99 \%)$ \\
\hline \multicolumn{2}{|l|}{ Washing frequency $(\mathrm{n}=253)$} \\
\hline$\bullet<1 \mathrm{x} /$ week & $26(10.3 \%)$ \\
\hline - 1-2x/week & $81(32.0 \%)$ \\
\hline - 3-4x/week & $108(42.7 \%)$ \\
\hline$\bullet>4 \mathrm{x} /$ week & $38(15.0 \%)$ \\
\hline Excessive sweating yes/no $(\mathrm{n}=240)$ & $29 / 211(12 / 88 \%)$ \\
\hline Use of hair product yes/no $(n=255)$ & $88 / 167(35 / 65 \%)$ \\
\hline - Mousse & $3(3.4 \%)$ \\
\hline - Hairspray & $6(6.9 \%)$ \\
\hline - Wax & $16(18.4 \%)$ \\
\hline - Gel & $23(26.4 \%)$ \\
\hline - Other & $26(30.0 \%)$ \\
\hline - Multiple & $13(14.9 \%)$ \\
\hline - missing & 7 \\
\hline Smoking yes/no $(\mathrm{n}=597)^{*}$ & 9/587 (2/98 \%) \\
\hline
\end{tabular}

Data described as mean (standard deviation) or number (percentage). Abbreviations: sd(s) standard deviation (score).

* In children who did not fill out a questionnaire, hair dyed was set to 'no' for children under the age of 10 years and current smoking status was set to 'no' under the age of 12 years.

different between the three age groups $(\mathrm{p}<0.001)$ and is higher in the older children. Due to limited hair amount we could only measure both hair cortisol and hair growth velocity in 47 of the 62 children. In these children, the lower cortisol levels during the first two years were partly explained by the increasing hair growth velocity. Log cortisol had a significant association with age $(\mathrm{p}<0.001)$; however, when hair growth velocity was taken into account, the association was no longer significant $(\mathrm{p}=0.252)$.

\subsection{EFFECTS OF DETERMINANTS ON CORTISOL MEASUREMENT}

Table 3 shows the effects of participant and hair related characteristics on cortisol z-scores. Hair cortisol was not associated with gender. Cortisol was lower in children of Western descent than in children of non-Western descent $(\mathrm{p}=0.04)$. All other known confounders (BMI, smoking, excessive sweating) were not associated with a difference in cortisol concentration. Hair care characteristics (washing frequency, hair product use, type of hair product and hair color) were not associated with hair cortisol level.

\section{Discussion}

This study is, to the best of our knowledge, the first to provide both LC-MS/MS-based reference intervals for cortisol concentrations in scalp hair of healthy children from birth to 18 years of age as well as hair growth rates in infants. Hair cortisol levels are age-dependent; remarkably high levels are observed after birth then much lower values in the first months followed by further lower values to a nadir at the age of 6 years. The higher cortisol levels that were found during infancy might be explained by both the lower hair growth rates found in children aged less than 2 years and by the different hair types in this age 


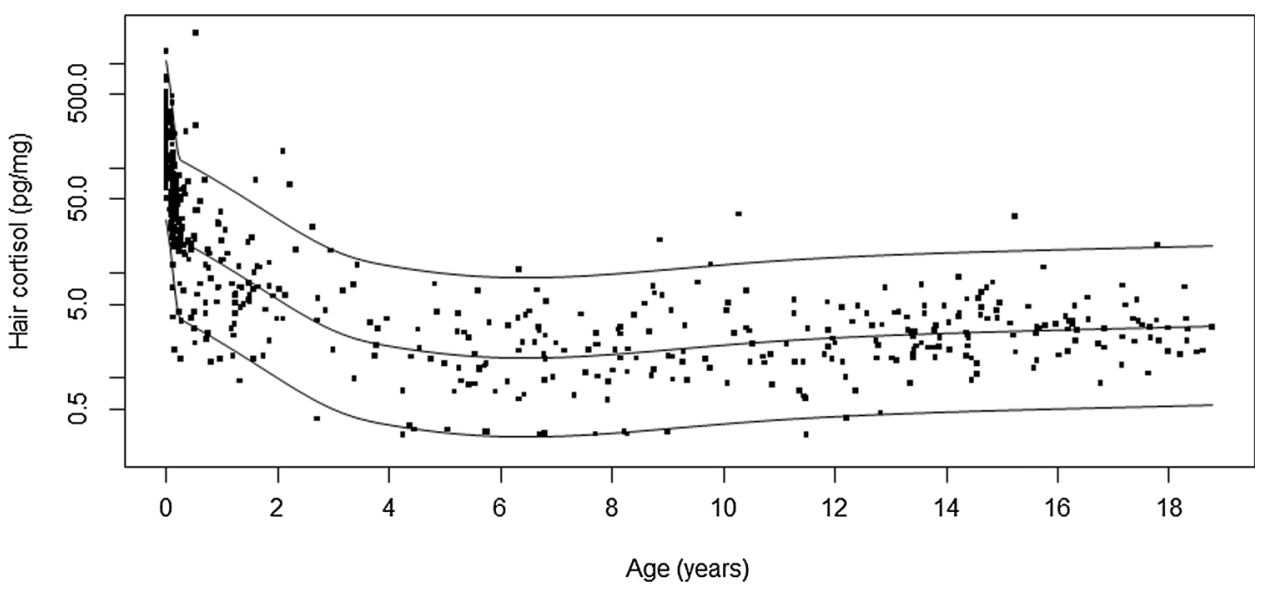

Fig. 1. Reference ranges cortisol concentrations over age.

Table 2

Age adjusted reference ranges for hair cortisol (pg/mg).

\begin{tabular}{|c|c|c|c|}
\hline Age & & Cortisol & \\
\hline Percentiles & 2.5 th & 50.0th & 97.5th \\
\hline at birth $(n=99)$ & 28.8 & 162.4 & 961.2 \\
\hline 1 month $(n=62)$ & 15.7 & 88.8 & 525.7 \\
\hline 2 months $(\mathrm{n}=71)$ & 6.82 & 38.5 & 227.8 \\
\hline 3 months $(n=24)$ & 3.64 & 20.5 & 121.5 \\
\hline 6 months $(n=30)$ & 2.84 & 16.0 & 94.9 \\
\hline 9 months $(n=13)$ & 2.42 & 13.7 & 80.9 \\
\hline 1 year $(n=15)$ & 2.05 & 11.6 & 68.6 \\
\hline 1.5 year $(n=25)$ & 1.45 & 8.20 & 48.6 \\
\hline 2 year $(n=14)$ & 1.06 & 5.73 & 33.9 \\
\hline 3 year $(n=11)$ & 0.56 & 2.91 & 17.2 \\
\hline 4 year $(n=10)$ & 0.34 & 1.93 & 11.4 \\
\hline 5 year $(n=15)$ & 0.29 & 1.65 & 9.79 \\
\hline 6 year $(n=20)$ & 0.27 & 1.55 & 9.14 \\
\hline 7 year $(n=26)$ & 0.28 & 1.56 & 9.23 \\
\hline 8 year $(n=13)$ & 0.30 & 1.67 & 9.85 \\
\hline 9 year $(n=21)$ & 0.32 & 1.83 & 10.8 \\
\hline 10 year $(n=10)$ & 0.36 & 2.02 & 12.0 \\
\hline 11 year $(n=20)$ & 0.39 & 2.21 & 13.1 \\
\hline 12 year $(n=9)$ & 0.42 & 2.38 & 14.1 \\
\hline 13 year $(n=23)$ & 0.45 & 2.51 & 14.9 \\
\hline 14 year $(n=25)$ & 0.47 & 2.63 & 15.5 \\
\hline 15 year $(n=18)$ & 0.48 & 2.73 & 16.2 \\
\hline 16 year $(n=20)$ & 0.50 & 2.82 & 16.7 \\
\hline 17 year $(n=14)$ & 0.52 & 2.91 & 17.2 \\
\hline 18 year $(n=17)$ & 0.53 & 3.00 & 17.8 \\
\hline
\end{tabular}

group, with variation in the cortex and the amount of medulla (Barth, 1987). This variation might have an effect on the incorporation of free cortisol into hair as this is thought to occur via passive diffusion from blood into the medulla of the hair shaft during growth (Russell et al., 2018).

The higher hair cortisol we found in older children is consistent with previous published literature that included adult participants. In a meta-analysis that included 10,289 individuals, the respective correlation coefficient was estimated to increase by 0.09 for each 10 -year increase in the standard deviation of age (Stalder et al., 2017). Consistent with this finding, we measured a median concentration of $3.0 \mathrm{pg} / \mathrm{mg}$ in 18-year-old children, while mean cortisol levels in adults range from 3.18 to $5.50 \mathrm{pg} / \mathrm{mg}$ (Abell et al., 2016; Sauvé et al., 2007; Staufenbiel et al., 2015)). A recent systematic review of the determinants of hair cortisol in children (Gray et al., 2018) analyzed age as a predictor of hair cortisol in children. Interestingly, in very young children, especially children under 2 years of age, cortisol was suggested to be particularly high (Dettenborn et al., 2012). Our study strongly confirms this finding.

Limited studies on reference intervals and potential confounders of cortisol concentration in children have been published (Dettenborn et al., 2012; Karlen et al., 2013; Rippe et al., 2016). Although only $25 \%$ of laboratories use LCMS technology (Gray et al., 2018), mass spectrometry has become the preferred method for steroid analysis in high-quality clinical research (Handelsman and Wartofsky, 2013). The laboratory of Erasmus MC already participated in the first international interlaboratory Round Robin in 2015 (Russell et al., 2015). Results from the Round Robin showed that immunoassay concentrations are higher by approximately a factor of 3 than the concentrations measured by LC-MS/MS. Therefore, we believe that our data can be interpreted well by colleagues using immunoassays.

Rippe et al. (Rippe et al., 2016) analyzed hair cortisol concentrations in a large cohort of 2484 Dutch children aged 6 years from the Generation R Study. Hair cortisol levels were associated with socioeconomic status, ethnicity, hair color and child characteristics such as birthweight, gestational age at birth, BMI, disease, allergy and medication use. The median cortisol concentrations of $1.55 \mathrm{pg} / \mathrm{mg}$ in boys and 1.38 in girls found by Rippe et al. correspond with the results found in the current study (1.55 at age 6 years). Two other studies found either comparable concentrations, Evans et al. (Evans et al., 2019) found that 10 -year old Dutch children had a median concentration of $2.99 \mathrm{pg} /$ $\mathrm{mg}$ (compared to 2.02 in our study), or fourfold higher hair cortisol values (Gao et al., 2014). Apart from the difference in ethnicity in the Gao study (participants were Chinese), both studies were similar in that they consisted of small numbers (92 and 29 participants) and did not report on potential confounding factors such as hair characteristics and corticosteroid use (Gray et al., 2018).

Our finding that hair cortisol, after infancy, is higher in older children, corresponds with previous data on traditional cortisol measurements in serum (Bailey et al., 2013; Wang et al., 2018). Serum cortisol concentrations steadily get higher from age 4 into adolescence. Kulle et al. (Kulle et al., 2013) reported age- and gender-specific reference data for serum cortisol in 905 children and reported high cortisol levels in the group aged less than 1 year. Although the current study measures cortisol concentrations in a different matrix, the associations with age resemble those in hormone studies performed in serum.

Several studies in adults have addressed the effects of potential confounders on cortisol concentrations measured in hair. The main covariates to be considered are gender (men exhibit $21 \%$ higher hair cortisol levels than women) (Stalder et al., 2017) and ethnicity (the highest median hair cortisol in blacks, followed by hispanics and whites (Abell et al., 2016; Wosu et al., 2015)). Rather weak associations are found with hair washing frequency, hair treatment and oral contraceptive use (Dettenborn et al., 2012; Gray et al., 2018; Manenschijn et al., 2011; Rippe et al., 2016; Sauvé et al., 2007). In accordance with these findings, we observed no major effects on hair cortisol concentrations due to hair characteristics but significant effects of 


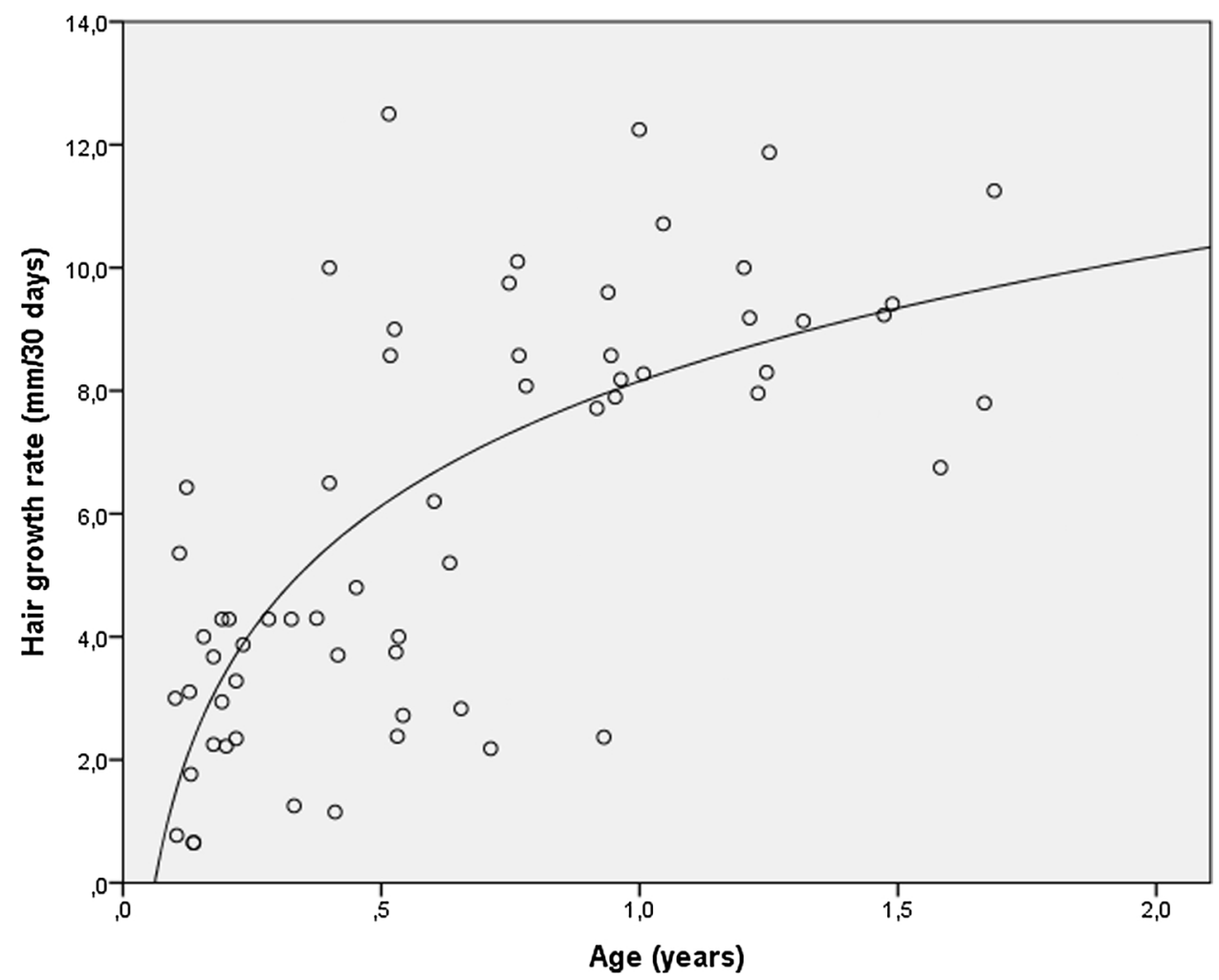

Fig. 2. Hair growth rate in relation to age.

Table 3

Associations with hair cortisol concentrations.

\begin{tabular}{|c|c|c|c|}
\hline & Beta & $\begin{array}{l}\text { Cortisol } \\
\text { SE }\end{array}$ & $\mathrm{P}$ \\
\hline \multicolumn{4}{|l|}{ Gender } \\
\hline Boys & Ref & & \\
\hline Girls & -0.07 & 0.09 & 0.431 \\
\hline \multicolumn{4}{|l|}{ Ethnicity } \\
\hline Western immigrant/Caucasian & Ref & & \\
\hline Nonwestern immigrant & 0.31 & 0.15 & 0.036 \\
\hline BMI sds & -0.01 & 0.07 & 0.907 \\
\hline Smoking: yes & -0.09 & 0.34 & 0.800 \\
\hline \multicolumn{4}{|l|}{ Hair color: } \\
\hline Brown & Ref & & \\
\hline Black & -0.17 & 0.28 & 0.546 \\
\hline Blond & 0.12 & 0.14 & 0.395 \\
\hline Red & -0.34 & 0.29 & 0.240 \\
\hline Hair treatment: yes & 0.11 & 0.24 & 0.658 \\
\hline \multicolumn{4}{|l|}{ Washing frequency: } \\
\hline$<1 \mathrm{x} /$ week & 0.41 & 0.22 & 0.060 \\
\hline 1-2x/week & 0.05 & 0.15 & 0.744 \\
\hline 3-4x/week & Ref & & \\
\hline$>4 \mathrm{x} /$ week & 0.15 & 0.19 & 0.441 \\
\hline Excessive sweating: yes & 0.24 & 0.20 & 0.218 \\
\hline Hair product use: yes & -0.04 & 0.13 & 0.778 \\
\hline
\end{tabular}

Beta's, standard error (SE) and p-values (P) of linear regression model. All models adjusted for gender and ethnicity. Hair cortisol is provided in z-scores adjusted for age.

ethnicity. Several explanations have been offered to explain this effect (Abell et al., 2016). First, certain ethnic groups are more likely to be exposed to biopsychosocial stressors (Boileau et al., 2019). Second, there are differences in the hair textures of different ethnic groups, and some textures may lead to more incorporation of cortisol into hair (Wosu et al., 2015). Third, hair grows at different rates in different ethnic groups (Loussouarn et al., 2016). Although the ethnic makeup of the newborn age group was different than in the other age groups, we think the difference between the very high cortisol levels after birth and the much lower values after the first months is not likely to be explained by ethnicity. In the whole cohort, the influence of ethnicity on cortisol levels was found to be significant but is subtle, within the normal range, compared to the extremely higher levels of cortisol in both western and non-western ethnic groups after birth. Finally, consistent with 11 of the 17 studies on determinants of hair cortisol in children reviewed by Gray (Gray et al., 2018), we found no significant difference in hair cortisol between girls and boys.

In our study, we noted a significantly lower growth rate of $3.5 \mathrm{~mm}$ per month in infants up to 6 months old, compared to $9.4 \mathrm{~mm} / \mathrm{month}$ in children aged 12-24 months and the generally accepted growth rate of $10,0 \mathrm{~mm}$ per month in adults (Saitoh et al., 1969). Our finding has important implications for the interpretation of hair cortisol values in very young children. There are several possible explanations for the lower growth rate. First, infant scalp hair consists of a different type of hair. In 1930, historical observations of 16 American white children using serial monthly hair samples from birth until the age of 14 years showed that the amount of medulla in newborn hair is much lower than in infant hair and increases into childhood (Duggins and Trotter, 1951). Barth (Barth, 1987) further described four types of infant hair: lanugo in newborns, vellus hair, an intermediate form of hair from between 3 and 7 months up to 2 years, followed by terminal, adult-like hair. We hypothesize that these four types of infant hair have different growth characteristics. Second, toward the end of the first year, the synchronized cyclic activity of all scalp hair follicles changes to a mosaic pattern; (Barth, 1987; Furdon and Clark, 2003). During fetal life and for at least the first four months post-birth, all scalp hair follicles are synchronized in the same phase of the growth cycle which consists of three phases: growth (anagen phase), cessation (catagen phase) and rest (telogen phase). This synchronization is lost near the end of the first year, resulting in differences in hair loss and in the percentage of terminal hair follicles on the scalp, which in turn leads to differences in the growth velocity of the hair present. There is considerable individual variation between ages at which the synchronization is lost, which probably explains the variation in individual growth rate from 0.7 to 
$12.5 \mathrm{~mm}$ that was found in our study in the age group $<12$-month age group. It is important to realize that it takes time for the growing hair to reach the surface. Third, ethnicity has a small, but significant effect on the individual growth rate: African hair grows more slowly than Caucasian hair, which in turn grows more slowly than Asian hair (mean \pm SD: $280 \pm 50,367 \pm 56$ and $411 \pm 43 \mu \mathrm{m} /$ day, respectively) (Loussouarn et al., 2016, 2005). As within the groups of immigrants in our study, western and non-western, both African and Asian hair types are represented, it is difficult to estimate the effect of ethnicity on the growth rate in our study.

Strengths of the present study include the use of a LC-MS/MS method in a representative population with multiple ethnicities. In the Netherlands $77 \%$ of the inhabitants are native Dutch and $23 \%$ are immigrants compared with 72.4 and $27.6 \%$ in our study (Statistics Netherlands, StatLine, CBS 2018). Next to this we measured a broad range of potential confounders. This is the first study to examine reference intervals across all age ranges from birth to 18 years including a large number of hair samples from the difficult-to-study young age group. For the first time, hair growth velocity has been studied in infants. A limitation in the current study is that immigrant ethnicity is defined as a dichotomous variable (Western vs. non-Western), which probably underrepresents the differences between ethnicities in the non-Western group. As ethnicity in the Netherlands is strongly associated with immigration, culture, different social economic status and education level (Flink et al., 2013), it is difficult to determine how ethnicity affects cortisol levels. Another limitation is that detailed information on demographic characteristics of the cohort, such as parental education, socioeconomic status, neighbourhood level advantage/ disadvantage is not available. Further reference studies in populations with different mixes of ethnicities and information on SES measures would be of additional value.

Measuring cortisol in scalp hair yields long-term cumulative cortisol concentrations unaffected by circadian rhythm or acute stress. The noninvasiveness of the procedure and easy storage of samples make this method exceptionally suitable for pediatric clinical practice. Longitudinal studies in which cortisol analysis in scalp hair are included may provide new insights into the pathophysiology of cortisol-related morbidity and leads to novel clinical applications for a range of endocrine diseases-including diagnosis and follow-up in hypercortisolism, adrenal insufficiency and congenital adrenal hyperplasia-as well as use in research and as a biomarker for stress and mental health.

This study provides knowledge about pediatric reference intervals and hair growth velocity in infants, which is essential for the implementation of hair cortisol testing and interpretation of the results in pediatric clinical practice.

\section{Funding}

This research was supported by the Elisabeth Foundation/Erasmus Trustfonds, a non-profit foundation supporting academic research.

\section{Disclosure statement}

The authors have nothing to disclose.

\section{Declaration of Competing Interest}

None

\section{Acknowledgments}

The authors are grateful to Ms. Margriet E. Bisschoff, research nurse, Erasmus MC, Rotterdam, The Netherlands, for her help in participant recruitment and measurements; to Ozair Abawi, MD/PhD student, for conversion of growth data to standard deviation scores; and to Carole Lasham for comments on the manuscript.

\section{Appendix A. Supplementary data}

Supplementary material related to this article can be found, in the online version, at doi:https://doi.org/10.1016/j.psyneuen.2019. 104539.

\section{References}

Abell, J.G., Stalder, T., Ferrie, J.E., Shipley, M.J., Kirschbaum, C., Kivimäki, M., Kumari, M., 2016. Assessing cortisol from hair samples in a large observational cohort: the Whitehall II study. Psychoneuroendocrinology 73, 148-156.

Bailey, D., Colantonio, D., Kyriakopoulou, L., Cohen, A.H., Chan, M.K., Armbruster, D. Adeli, K., 2013. Marked biological variance in endocrine and biochemical markers in childhood: establishment of pediatric reference intervals using healthy community children from the CALIPER cohort. Clin. Chem. 59, 1393-1405.

Barth, J.H., 1987. Normal hair growth in children. Pediatr. Dermatol. 4, 173-184.

Boileau, K., Barbeau, K., Sharma, R., Bielajew, C., 2019. Ethnic differences in diurnal cortisol profiles in healthy adults: a meta-analysis. Br. J. Health Psychol. 1-22.

Dettenborn, L., Tietze, A., Kirschbaum, C., Stalder, T., 2012. The assessment of cortisol in human hair: associations with sociodemographic variables and potential confounders. Stress 15, 578-588.

Evans, B.E., Beijers, R., Hagquist, C., de Weerth, C., 2019. Childhood urbanicity and hair steroid hormone levels in ten-year-old children. Psychoneuroendocrinology 102 53-57.

Flink, I.J.E., Beirens, T.M.J., Looman, C., Landgraf, J.M., Tiemeier, H., Mol, H.A., Jaddoe, V.W.V., Hofman, A., Mackenbach, J.P., Raat, H., 2013. Health-related quality of life of infants from ethnic minority groups: the Generation R Study. Qual. Life Res. 22, 653-664.

Furdon, S.A., Clark, D.A., 2003. Scalp hair characteristics in the newborn infant. Adv. Neonatal Care 3, 286-296.

Gao, W.E.I., Zhong, P., Xie, Q., Wang, H., Jin, J., Deng, H., Lu, Z., 2014. Temporal features of elevated hair cortisol among earthquake survivors. Psychophysiology 51, 319-326.

Gray, N.A., Dhana, A., Van Der Vyver, L., Van Wyk, J., Khumalo, N.P., Stein, D.J., 2018. Determinants of hair cortisol concentration in children: a systematic review. Psychoneuroendocrinology 87, 204-214.

Greff, M.J.E., Levine, J.M., Abuzgaia, A.M., Elzagallaai, A.A., Rieder, M.J., van Uum, S.H.M., 2018. Hair cortisol analysis: an update on methodological considerations and clinical applications. Clin. Biochem. 63, 1-9.

Handelsman, D.J., Wartofsky, L., 2013. Requirement for mass spectrometry sex steroid assays in the journal of clinical endocrinology and metabolism. J. Clin. Endocrinol. Metab. 98, 3971-3973.

Hodes, A., Meyer, J., Lodish, M.B., Stratakis, C.A., Zilbermint, M., 2018. Mini-review of hair cortisol concentration for evaluation of Cushing syndrome. Expert Rev. Endocrinol. Metab. 13, 225-231.

Hollanders, J.J., van der Voorn, B., Kieviet, N., Dolman, K.M., de Rijke, Y.B., van den Akker, E.L.T., Rotteveel, J., Honig, A., Finken, M.J.J., 2017. Interpretation of glucocorticoids in neonatal hair: a reflection of intrauterine glucocorticoid regulation? Endocr. Connect. 6, 692-699.

Ibrahim, C., Van Uum, S., 2014. Hair analysis of cortisol levels in adrenal insufficiency. CMAJ 186, 1244.

Karlen, J., Frostell, A., Theodorsson, E., Faresjo, T., Ludvigsson, J., 2013. Maternal influence on child HPA axis: a prospective study of cortisol levels in hair. Pediatrics 132, e1333-40.

Kieviet, N., de Groot, S., Noppe, G., de Rijke, Y.B., van Rossum, E.F.C., van den Akker, E.L.T., Dolman, K.M., Honig, A., 2016. Is poor neonatal adaptation after exposure to antidepressant medication related to fetal cortisol levels? An explorative study. Early Hum. Dev. 98, 37-43.

Kulle, A.E., Welzel, M., Holterhus, P.M., Riepe, F.G., 2013. Implementation of a liquid chromatography tandem mass spectrometry assay for eight adrenal C-21 steroids and pediatric reference data. Horm. Res. Paediatr. 79, 22-31.

Loussouarn, G., Lozano, I., Panhard, S., Collaudin, C., El Rawadi, C., Genain, G., 2016. Diversity in human hair growth, diameter, colour and shape. An in vivo study on young adults from 24 different ethnic groups observed in the five continents. Eur. J. Dermatol. 26, 44-54.

Loussouarn, G., Rawadi, C.E., Genain, G., 2005. Original article Diversity of hair growth profiles. Int. J. Dermatol. 44, 6-9.

Manenschijn, L., Koper, J.W., Lamberts, S.W.J., Van Rossum, E.F.C., 2011. Evaluation of a method to measure long term cortisol levels. Steroids. 76, 1032-1036.

Manenschijn, L., Schaap, L., Van Schoor, N.M., Van Der Pas, S., Peeters, G.M.E.E., Lips, P., Koper, J.W., Van Rossum, E.F.C., 2013. High long-term cortisol levels, measured in scalp hair, are associated with a history of cardiovascular disease. J. Clin. Endocrinol. Metab. 98, 2078-2083.

Noppe, G., De Rijke, Y.B., Dorst, K., Van Den Akker, E.L.T., Van Rossum, E.F.C., 2015. LCMS/MS-based method for long-term steroid profiling in human scalp hair. Clin. Endocrinol. (Oxf). 83, 162-166.

Noppe, G., Van Den Akker, E.L.T., De Rijke, Y.B., Koper, J.W., Jaddoe, V.W., Van Rossum, E.F.C., 2016. Long-term glucocorticoid concentrations as a risk factor for childhood obesity and adverse body-fat distribution. Int. J. Obes. 40, 1503-1509.

Noppe, G., Van Rossum, E.F.C., Vliegenthart, J., Koper, J.W., Van Den Akker, E.L.T., 2014. Elevated hair cortisol concentrations in children with adrenal insufficiency on hydrocortisone replacement therapy. Clin. Endocrinol. (Oxf). 81, 820-825.

Papafotiou, C., Christaki, E., van den Akker, E.L.T., Wester, V.L., Apostolakou, F., 
Papassotiriou, I., Chrousos, G.P., Pervanidou, P., 2017. Hair cortisol concentrations exhibit a positive association with salivary cortisol profiles and are increased in obese prepubertal girls. Stress 20, 217-222.

Pecoraro, V., Astore, I., Barman, J., Ignacioaraujo, C., 1964. The normal trichogram in the child before the age of puberty. J. Invest. Dermatol. 42, 427-430.

Rigby, R.A., Stasinopoulos, D.M., Lane, P.W., 2005. Generalized additive models for location, scale and shape. J. R. Stat. Soc. Ser. C Appl. Stat. 54, 507-554.

Rippe, R.C.A., Noppe, G., Windhorst, D.A., Tiemeier, H., Van Rossum, E.F.C., Jaddoe, V.W.V., Verhulst, F.C., Bakermans-kranenburg, M.J., Van Ijzendoorn, M.H., Van Den Akker, E.L.T., 2016. Psychoneuroendocrinology splitting hair for cortisol? Associations of socio-economic status, ethnicity, hair color, gender and other child characteristics with hair cortisol and cortisone. Psychoneuroendocrinology 66 , 56-64.

Russell, E., Kirschbaum, C., Laudenslager, M.L., Stalder, T., De Rijke, Y., Van Rossum, E.F.C., Van Uum, S., Koren, G., 2015. Toward standardization of hair cortisol measurement: results of the first international interlaboratory round robin. Ther. Drug Monit. 37, 71-75.

Russell, E., Koren, G., Rieder, M., Van Uum, S., 2018. Hair cortisol as a biological marker of chronic stress : current status, future directions and unanswered questions. Psychoneuroendocrinology 37, 589-601.

Saitoh, M., Uzuka, M., Sakamoto, M., 1969. Rate of hair growth. Advances in Biology of Skin Vol. IX. Hair Growth.

Sauvé, B., Koren, G., Walsh, G., Tokmakejian, S., Van Uum, S.H.M., 2007. Measurement of cortisol in human hair as a biomarker of systemic exposure. Clin. Invest. Med. 30, E183-91.

Savas, M., Wester, V., de Rijke, Y.B., Rubinstein, G., Zopp, S., Dorst, K., van den Berg, S., Beuschlein, F., Feelders, R.A., Reincke, M., van Rossum, E.F.C., 2019. Hair glucocorticoids as biomarker for endogenous Cushing's syndrome: validation in two independent cohorts. Neuroendocrinology 109, 171-178.

Stalder, T., Kirschbaum, C., Alexander, N., Bornstein, S.R., Gao, W., Miller, R., Stark, S., Bosch, J.A., Fischer, J.E., 2013. Cortisol in hair and the metabolic syndrome. J. Clin. Endocrinol. Metab. 98, 2573-2580.

Stalder, T., Steudte-schmiedgen, S., Alexander, N., Klucken, T., Vater, A., Wichmann, S. Kirschbaum, C., Miller, R., 2017. Stress-related and basic determinants of hair cortisol in humans: a meta-analysis. Psychoneuroendocrinology 77, 261-274.

Staufenbiel, S.M., Penninx, B.W.J.H., de Rijke, Y.B., van den Akker, E.L.T., van Rossum, E.F.C., 2015. Determinants of hair cortisol and hair cortisone concentrations in adults. Psychoneuroendocrinology 60, 182-194.

Staufenbiel, S.M., Penninx, B.W.J.H., Spijker, A.T., Elzinga, B.M., van Rossum, E.F.C., 2013. Hair cortisol, stress exposure, and mental health in humans: a systematic review. Psychoneuroendocrinology 38, 1220-1235.

Vliegenthart, J., Noppe, G., van Rossum, E.F.C., Koper, J.W., Raat, H., van den Akker, E.L.T., 2016. Socioeconomic status in children is associated with hair cortisol levels as a biological measure of chronic stress. Psychoneuroendocrinology 65, 9-14.

Wang, L., Zhang, L., Wang, H., Wen, H., Tao, H., Zhao, X., 2018. Chinese pediatric reference intervals for serum cortisol on IMMULITE 2000. Ann. Lab. Med. 38, 59-62.

Wester, V.L., Noppe, G., Savas, M., van den Akker, E.L.T., de Rijke, Y.B., van Rossum, E.F.C., 2017. Hair analysis reveals subtle HPA axis suppression associated with use of local corticosteroids: the Lifelines cohort study. Psychoneuroendocrinology 80, 1-6.

Wester, V.L., Van Rossum, E.F.C., 2015. Clinical applications of cortisol measurements in hair. Eur. J. Endocrinol. 173, M1-M10.

Wosu, A.C., Gelaye, B., Valdimarsdóttir, U., Kirschbaum, C., Stalder, T., Shields, A.E., Williams, M.A., 2015. Hair cortisol in relation to sociodemographic and lifestyle characteristics in a multiethnic US sample. Ann. Epidemiol. 25, 90-95. 